\title{
Mass Spectrometry Characterization of the Glycation Sites of Bovine Insulin by Tandem Mass Spectrometry
}

\author{
Sofia Guedes, Rui Vitorino, M. Rosário M. Domingues, \\ Francisco Amado, and Pedro Domingues \\ Department of Chemistry, University of Aveiro, Aveiro, Portugal
}

Bovine insulin was glycated under hyperglycemic reducing conditions and in nonreducing conditions. Purification through HPLC allowed isolating glycated forms of insulin and a novel triglycated form (6224.5 Da) was purified. Endoproteinase Glu-C digestion combined with mass spectrometry (MALDI-TOF/TOF) allowed determining the exact location of the glycation sites in each of the isolated glycated insulins. For the first time, a triglycated form of insulin was isolated and characterized accordingly to its glycation sites. These glucose binding sites were identified as the N-terminals of both chains (Gly1 and Phe1) and residue Lys29 of B-chain. Moreover, in diglycated insulin we found the coexistence of one specie glycated at the N-terminals of both chains (Gly1 and Phe1) and another specie containing the two glucitol adducts in B-chain (Phe1 and Lys29). Also, in monoglycated insulin generated in reducing and nonreducing conditions, one specie glycated at Phe1 and another specie glycated at Lys29, both B-chain residues coexist. (J Am Soc Mass Spectrom 2009, 20, 1319-1326) (C) 2009 American Society for Mass Spectrometry

$\mathrm{O}$ ver the last years, particular attention has been given to the role of nonenzymatic glycation in diabetic complications development, including neuropathy, nephropathy, retinopathy, and cardiovascular disease [1]. Under conditions of hyperglycemia, glycation compromises several functional proteins, such as hemoglobin $[2,3]$, glucose-6-phosphate dehydrogenase $[4,5]$, aldehyde reductase [6], glutathione reductase [7, 8], glutathione peroxidase [8,9], and $\mathrm{Cu}-\mathrm{Zn}$ superoxide dismutase $[10,11]$. In this context, insulin glycation is of particular interest. The structurally modified peptide exhibits decreased ability to regulate plasma glucose homeostasis and is associated with reduced hepatic clearance, decreased adipose tissue lipogenesis, and a decreased glucose uptake and oxidation in isolated diaphragm and abdominal muscle in vitro [12-15]. Even though the short half-life of insulin (5-10 $\mathrm{min})$, experiments performed to date indicate that a substantial proportion of insulin (and proinsulin) is glycated in the pancreatic cells during the stages of insulin synthesis and storage [16]. Glycated insulin has been measured in the pancreas of various animal models of type 2 diabetes [17], and in both isolated islets and clonal $\beta$-cells exposed to elevated glucose concentrations in tissue culture [18, 19]. The significant compromised biological activity of glycated insulin raises the possi-

Address reprint requests to Professor Pedro Domingues, Department of Chemistry, University of Aveiro, 3810-193 Aveiro, Portugal. E-mail: p.domingues@ua.pt bility of its contribution to insulin resistance and glucose intolerance of type 2 diabetes.

Earlier studies indicated that during glycation process, each insulin molecule can bind to $\sim$ three to eight molecules of glucose [14, 20]. More recently, independent reports generalized the view that there are three possible free amino groups in the insulin molecule, human or bovine, available for in vitro glucose binding [15, 21-23]. Nowadays, through ESI-MS/MS and MALDI-MS approaches, it is established the N-terminal Phe1 residue of the B-chain is the only glycation site of human [23] and bovine [21] insulin. Moreover, O'Harte and coworkers determined, in a novel diglycated insulin (under hyperglycemic reducing conditions), the Nterminals Gly1 of A-chain and Phe1 of B-chain as the sites of glycation for human insulin [15], after Glu-C enzymatic digestion and identification of digested fragments by plasma desorption mass spectrometry (PDMS). Since human and bovine insulin only differ in three residues (A chain: residues 8 and 10; $\mathrm{B}$ chain: residue $30)$, it is expected that glycation of bovine insulin should occur in similar positions.

The present study structurally characterizes three forms of glycated bovine insulin when glycated under reducing conditions. Simultaneously, the characterization of glycated insulin produced under pseudophysiological conditions was also done. Our approach included HPLC purification of mono-, di-, and triglycated insulin forms, followed by enzymatic digestion and mass spectrometry (MALDI-TOF/TOF) for the unambiguous assignment of the glycation sites. 


\section{Materials and Methods}

\section{Chemicals and Reagents}

Bovine insulin (I5500) and TFA were acquired from Sigma (St. Louis, MO). The MALDI matrices $\alpha$-cyano4-hydroxy cinnamic acid (CHCA) and sinapinic acid were supplied by Merck (Darmstadt, Germany). The organic solvents such as toluene and acetonitrile (ACN) were from Riedel-deHaën (Buchs, Switzerland) and Labscan (Dublin, Ireland), respectively.

All chemicals were of analytical grade and milli-Q water (Millipore, Bedford, MA) was used throughout all experiments.

\section{Insulin In Vitro Glycation}

In vitro glycation was carried out by incubating insulin with D-glucose in a protocol adapted from O'Harte et al. where reducing [23] and nonreducing conditions were used. In nonreducing conditions, insulin $(200 \mu \mathrm{g}$ in $\mathrm{HCl} 2 \mathrm{mM}$ ) was incubated with D-glucose $220 \mathrm{mM}$ in phosphate buffer $10 \mathrm{mM}(\mathrm{pH} 7.4)$ at $37^{\circ} \mathrm{C}$ for $30 \mathrm{~d}$ (toluene $5 \mathrm{mM}$ was added as a bacteriostatic). In reducing conditions and according to the authors, insulin $(100 \mu \mathrm{g}$ in $\mathrm{HCl} 2 \mathrm{mM})$ was incubated for $24 \mathrm{~h}$ at $37^{\circ} \mathrm{C}$ with D-glucose $220 \mathrm{mM}$ (prepared in phosphate buffer $10 \mathrm{mM}, \mathrm{pH}$ 7.4) together with a 1000-fold molar excess of the reducing agent $\mathrm{NaBH}_{3} \mathrm{CN}$ (stock in ice cold $\mathrm{NaOH} 10 \mathrm{mM}$ ) in phosphate buffer $10 \mathrm{mM}$ (pH 7.4). In both cases, after incubation, reaction was stopped by addition of acetic acid $0.5 \mathrm{M}$, and the reaction mixture was dialyzed overnight against water (cut-off $1000 \mathrm{Da}$ ). Glycated and native forms were separated on a C-8 analytical column (Acclaim, $4.6 \times 250 \mathrm{~mm}, 5 \mu \mathrm{m}$ particle size; Dionex, Sunnyvale, CA) at a flow rate of $0.8 \mathrm{~mL} / \mathrm{min}$. The program was as follows: linear gradient of $0 \%$ to $35 \%$ ACN over $10 \mathrm{~min}$, followed by $35 \%$ to $56 \% \mathrm{ACN}$ over 20 min, and $56 \%$ to $70 \%$ ACN over 5 min. Peaks were hand-collected, pooled from several runs, and concentrated on a Speed-vac. Re-injection of each glycated form using the same separation program allowed to improve the purification of each glycated insulin form. This purification was monitored through MALDI-MS.

\section{Enzymatic Digestion (Endoproteinase Glu-C)}

For in-solution digestion of glycated and native insulin, samples were initially reconstituted in $\mathrm{HCl} 2 \mathrm{mM}$. Quantification was performed using the Quant-iT Protein Assay kit (Invitrogen, Paisley, UK) combined with the Qubit fluorometer. An aliquot was taken and diluted for enzymatic digestion with phosphate buffer $10 \mathrm{mM} \mathrm{pH}$ 7.8; $\mathrm{pH}$ is determinant since the specificity of endoproteinase Glu-C for glutamic acid depends on this parameter. Endoproteinase Glu-C (Calbiochem, Merck, Darmstadt, Germany) solution was added in a final ratio of substrate: enzyme of 20:1 (wt/wt). Duplicate samples were incubated overnight at $25^{\circ} \mathrm{C}$. After digestion, samples were dried under vacuum in a Speed-vac and, unless taken for analysis, stored at $-80^{\circ} \mathrm{C}$.

\section{Reduction of Disulphide Bridges of Insulin}

The disulphide bridges of monoglycated, diglycated, triglycated, and native insulin were reduced using DTT $10 \mathrm{mM}$ in phosphate buffer $\mathrm{pH} 8$ for $1 \mathrm{~h}$ at $55^{\circ} \mathrm{C}$. An aliquot of each sample $(10 \mu \mathrm{L})$ was taken and acidified with TFA $1 \%(1 \mu \mathrm{L})$. These aliquots were cleaned up using ZipTip C18 (Millipore, Bedford, MA) before mass spectrometry analysis.

\section{Mass Spectrometry}

Glu-C digests of glycated and native insulin were reconstituted in TFA $0.1 \%$. An aliquot of each sample was mixed (1:1) with $\alpha$-CHCA matrix solution $(5 \mathrm{mg} / \mathrm{mL}$ in ACN 50\%/TFA 0.1\%) and applied onto 384-well MALDI plates. Instrument was calibrated by adding insulin (12.5 ng; $0.5 \mu \mathrm{L}$ ) to each calibration spot. Peptide mass spectra were obtained with a MALDI-TOF/TOF mass spectrometer (4800 Proteomics Analyzer; Applied Biosystems, Foster (ity, CA) in the positive ion reflector mode. Spectra were obtained in the mass range between 800 and 7000 Da. Manual interpretation of tandem mass spectra was performed through the Data Explorer software TM ver. 4.4 (Applied Biosystems).

\section{Results and Discussion}

Glycation of insulin was monitored by MALDI-MS through the increase in the molecular weight of insulin as a result of glucose adducts formation with the hormone. Figure 1 shows the typical mass spectra of native (a) and glycated insulin (b and c). In Figure 1b, we can observe a typical spectrum of glycated insulin where glycation of the hormone was performed by incubation with $220 \mathrm{mM}$ glucose for $30 \mathrm{~d}$ at $37^{\circ} \mathrm{C}$ in the absence of reducing conditions. The major peak at $\mathrm{m} / \mathrm{z}$ 5730.66 corresponds to native bovine insulin (theoreti$\mathrm{cal} \mathrm{m} / \mathrm{z}$ 5730.6) whereas the second peak at $\mathrm{m} / \mathrm{z} 5892.72$ with a mass shift of 162 Da corresponds to a monoglycated form of insulin. A third minor peak at $\mathrm{m} / \mathrm{z} 6054.71$ is also observable and should correspond to a diglycated form with a mass shift of $324 \mathrm{Da}$. Figure 1c, where insulin glycation was performed by incubation with 220 $\mathrm{mM}$ glucose for $30 \mathrm{~h}$ at $37^{\circ} \mathrm{C}$ in reducing conditions $\left(\mathrm{NaBH}_{3} \mathrm{CN}\right)$, shows a different mass profile, especially in terms of relative abundance of each specie. There are three additional peaks in the spectrum besides the peak corresponding to native insulin. The major peak, at $\mathrm{m} / \mathrm{z}$ of 5894.72, is the protonated monoglycated form with a mass shift of $164 \mathrm{Da}$ (glucitol adduct). The two other peaks at $\mathrm{m} / \mathrm{z} 6058.89$ and 6222.82 correspond to the protonated diglycated and triglycated forms, respectively. With the mono-, di-, and triglycated forms, several additional peaks are observed. These peaks are BH3-adducts $(\Delta \mathrm{m}=14 \mathrm{Da})$, resulting from the in vitro 

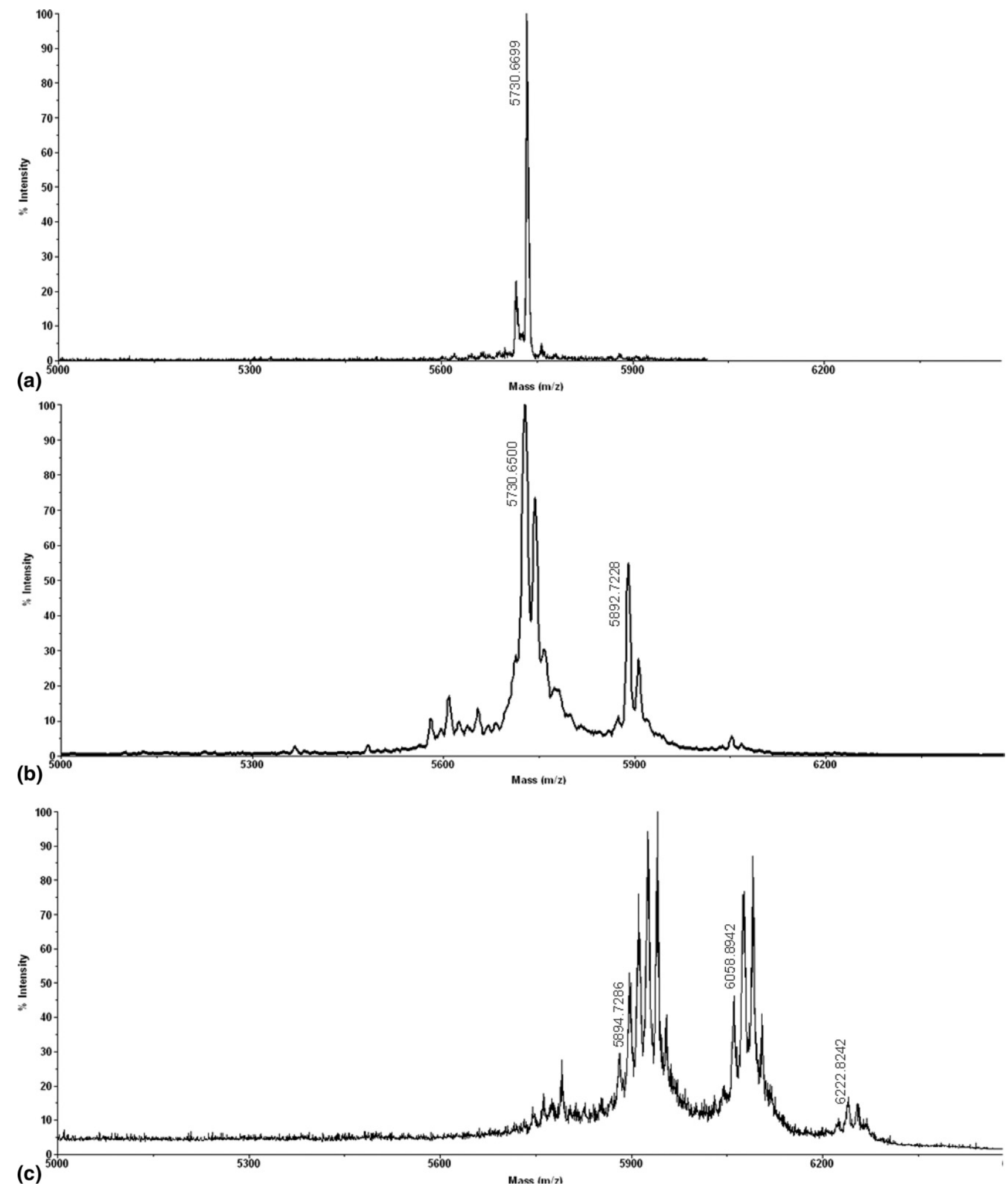

Figure 1. Typical MALDI-MS spectra of native insulin and glycated insulin in both reducing and nonreducing conditions. (a) Native insulin, (b) glycated insulin under nonreducing conditions, (c) glycated insulin under reducing conditions.

glycation procedure where an excess of this reducing agent is used.

After separating the different glycated forms of insulin by HPLC-C8, purified samples were reconstituted in $\mathrm{HCl} 2 \mathrm{mM}$ and quantified with Quant-iT Protein Assay kit. In the absence of reducing conditions, 32\% of the purified monoglycated form was obtained. In reducing conditions $45 \%$ of monoglycated, $25 \%$ of di- 
glycated, and $6 \%$ of triglycated forms of insulin were obtained.

To identify the glycation sites, each sample was enzymatically digested with endoproteinase Glu-C as described in the experimental section. Cleavage of insulin at glutamic acid residues without disulfide bridges disruption theoretically produces nine fragments (Table 1 ). Digested samples of glycated and native insulin were analyzed by MALDI-MS, and Figure 2 shows representative spectrum of Glu-C digests for all studied samples. Highlighted peaks that are present solely in glycated samples were chosen for analysis through tandem mass spectrometry (MALDI-MS/MS). Table 1 shows the identified peaks in all samples. Figure 3 shows the two MS/MS spectra of the peptides at $\mathrm{m} / \mathrm{z} 1248.57$ (Figure 3a) and 1644.68 (Figure 3b), identified as fragments B1-13 and B22-30 from glycated insulin (no reducing conditions) with one glucose adduct attached (see Table 1).

Analysis of the MS/MS spectrum of the modified peptide at $\mathrm{m} / \mathrm{z} 1248.57$ (Figure 3a) allows locating the glucose adduct in residue Lys29. In the MS/MS spectrum, it is possible to see the mass difference of $290.2 \mathrm{Da}$ between the ion at $\mathrm{m} / \mathrm{z} 1131.5$ (a8) and the ion at $\mathrm{m} / \mathrm{z}$ 841.3 (a7). This gap corresponds to the loss of the lysine residue with a glucose adduct. Analysis of the MS/MS spectrum of the precursor ion at 1644.68 (see Figure 3b) allows locating the glucose adduct in residue Phe1. These results agree with the literature [21, 22], which state the most likely sites of glycation within the B-chain are the Phe1 and Lys 29 residues, as both contain free amino groups for linking glucose molecules. Nevertheless, the preferred glycation site of monoglycated bovine or human insulin is thought to be at Phe-1 of the

Table 1. Theoretical peptide fragments from endoproteinase Glu-C digestion of native insulin and experimental glycated fragments obtained from Glu-C digests of glycated insulin samples. Underlined residues correspond to cysteines involved in disulfide bridges

\begin{tabular}{|c|c|c|c|c|}
\hline Position & Peptide sequence & $\begin{array}{l}\text { Theoretical } \\
\text { mass }(\mathrm{Da})\end{array}$ & $\begin{array}{l}\text { Experimental } \\
\text { mass }(\mathrm{Da})\end{array}$ & $\begin{array}{l}\text { Match } \\
\text { error }(\mathrm{Da})\end{array}$ \\
\hline \multicolumn{5}{|l|}{ Native insulin } \\
\hline A $1-4$ & GIVE & 417.2324 & - & - \\
\hline A $5-17$ & QCCCASV́SLYQLE & 1446.6062 & - & - \\
\hline A $5-17$ & 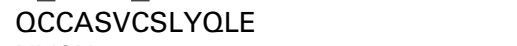 & 1444.5906 & 1444.4225 & -0.1681 \\
\hline A $18-21$ & NYCN & 513.1762 & - & - \\
\hline B 1-13 & FVNOHLCGSHLVE & 1482.7158 & 1482.6351 & -0.0807 \\
\hline B $14-21$ & ALYLVCGE & 867.4280 & 867.4041 & -0.0239 \\
\hline B 22-30 & RGFFYTPKA & 1086.5731 & 1086.5048 & -0.0683 \\
\hline A $5-17 / B$ 1-13 & QCCASVCSLYQLE/FVNOHLCGSHLVE & 2924.283 & - & - \\
\hline A $18-21 / B$ 14-21 & $N \overline{Y C} N / A L \bar{Y} L V \underline{C G E}$ & 1377.5808 & 1377.4717 & -0.1091 \\
\hline \multicolumn{5}{|l|}{ Glycated insulin } \\
\hline \multicolumn{5}{|c|}{$\begin{array}{l}\text { Monoglycated insulin (nonreducing } \\
\text { conditions) }\end{array}$} \\
\hline B $14-21$ & ALYLVCGE & 867.4280 & 867.4378 & 0.0098 \\
\hline B 22-30 & RGFFYTPKA & 1086.5731 & 1086.5291 & -0.0440 \\
\hline B $22-30$ & RGFFYTPKA & 1248.6259 & 1248.5710 & -0.0549 \\
\hline A $18-21 / B$ 14-21 & NYCCN/ALYLVㅁGE & 1377.5808 & 1377.5029 & -0.0779 \\
\hline A $5-17$ & 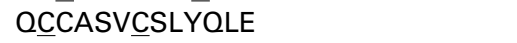 & 1444.5906 & 1444.5505 & -0.0401 \\
\hline B 1-13 & FVNNOHLCGSHLVE & 1482.7158 & 1482.6345 & -0.0813 \\
\hline B $1-13$ & FVNOHLCGSHLVE & 1644.7686 & 1644.6820 & -0.0866 \\
\hline \multicolumn{5}{|c|}{ Glycated insulin (reducing conditions) } \\
\hline \multicolumn{5}{|c|}{ Monoglycated } \\
\hline B 22-30 & RGFFYTPKA & 1086.5731 & 1086.4946 & -0.0785 \\
\hline B $22-30$ & RGFFYTPKA & 1250.6415 & 1250.5546 & -0.0869 \\
\hline A $18-21 / B$ 14-21 & NYCNN/ALYLVㅁGE & 1377.5808 & 1377.4662 & -0.1146 \\
\hline A $5-17$ & 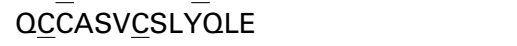 & 1444.5906 & 1444.5294 & -0.0612 \\
\hline B $1-13$ & FVNOHLCGSHLVE & 1646.7842 & 1646.6748 & -0.1094 \\
\hline \multicolumn{5}{|l|}{ Diglycated } \\
\hline B 22-30 & RGFFYTPKA & 1086.5731 & 1086.4970 & -0.0761 \\
\hline B 22-30 & RGFFYTPKA & 1250.6415 & 1250.5597 & -0.0818 \\
\hline A $18-21 / B \quad 14-21$ & 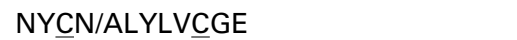 & 1377.5808 & 1377.4740 & -0.1068 \\
\hline A $5-17$ & QCECASVĆSLYQLE & 1444.5906 & 1444.5748 & -0.0158 \\
\hline B $1-13$ & FVNOHLCGSHLVE & 1646.7842 & 1646.7093 & -0.0749 \\
\hline A $1-21$ & GIVEOCCASVCSLYQLENYCN & 2501.0345 & 2501.0765 & 0.0420 \\
\hline \multicolumn{5}{|l|}{ Triglycated } \\
\hline B 22-30 & RGFFYTPKA & 1086.5731 & 1086.4949 & -0.0782 \\
\hline B $22-30$ & RGFFYTPKA & 1250.6415 & 1250.5478 & -0.0937 \\
\hline A $18-21 / B \quad 14-21$ & NYCN/ALYLVCGE & 1377.5808 & 1377.4398 & -0.1410 \\
\hline A $5-17$ & QCECASVĆSLYQLE & 1444.5906 & 1444.5100 & -0.0806 \\
\hline B $1-13$ & FVNOHLCGSHLVE & 1646.7842 & 1646.6378 & -0.1464 \\
\hline A $1-21$ & GIVEQCCCASVĆSLYQLENYCN & 2501.0345 & 2501.1065 & 0.0720 \\
\hline
\end{tabular}




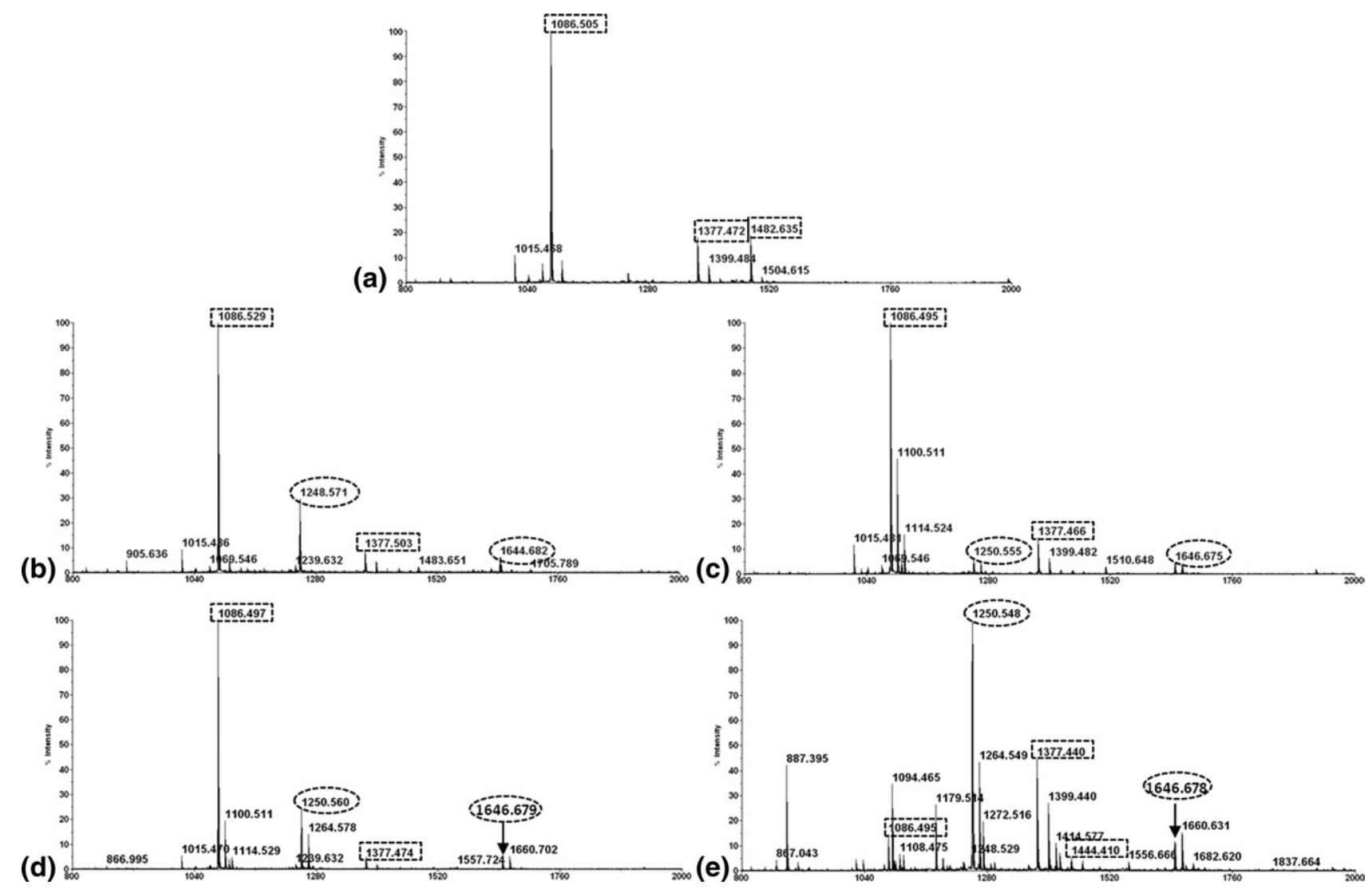

Figure 2. MALDI-MS spectra of Glu-C digests of native insulin and isolated forms of glycated insulin for both glycation procedures. The $\mathrm{m} / \mathrm{z}$ values of fragments matching theoretical peptide fragments shown in Table 1 are highlighted with rectangles. Peptide fragments highlighted with circles in glycated samples are unique to these samples and correspond to glycated fragments. (a) Native insulin, (b) monoglycated insulin under nonreducing conditions, (c) monoglycated insulin under reducing conditions, (d) diglycated insulin (reducing conditions), (e) triglycated insulin (reducing conditions).

B-chain [23]. This is most probably due to the high reactivity as this residue is situated at the N-terminal of the peptide chain. Herein, we show that monoglycated insulin produced in the absence of reducing conditions comprises the coexistence of two glycated insulin species with two different glycation sites, Phe1 or Lys29.

Figure 4 shows three representative examples of MS/MS spectra of glycated fragments belonging to glycated insulin produced under reducing conditions. The two glycated fragments that result from Glu-C digestion are the peptides at $\mathrm{m} / \mathrm{z} 1250.56$ and $\mathrm{m} / \mathrm{z}$ 1646.67 , and were found in all of three forms of glycated insulin (see Figure 2). These peptides were fragments B1-13 and B22-30 with identical glycation sites, Phe1 and Lys29, respectively, as reported for the monoglycated insulin formed in the absence of reducing conditions. In this case, because of the excess of reducing agent $\left(\mathrm{NaBH}_{3} \mathrm{CN}\right)$, the mass shift found in the MS/MS spectra corresponds to the attachment of a glucitol adduct (164 Da). Again, the coexistence of two glycated forms occurs in monoglycated insulin produced under reducing conditions.

With the approach described so far, it was not possible to identify any glycated fragments belonging to the A-chain in monoglycated, diglycated and triglycated forms of insulin. To induce the separation of the two chains (A and B) of the insulin samples, the disulphide bridges were reduced with DTT using an aliquot of undigested samples, as described in the Experimental section. These reduced samples were submitted to MALDI-MS and MALDI-MS/MS analysis. Figure 4c shows the representative MS/MS spectrum of the A-chain containing a single glucitol adduct, with an $\mathrm{m} / \mathrm{z} 2501$, which was found only in diglycated and triglycated forms of insulin. The identification of the complete $b$ series allowed the identification of Gly1 as the glycation site in the A-chain. This result allowed determining the three sites of glycation in triglycated insulin and also pointed out the presence of two isoforms in diglycated insulin, similar to the monoglycated forms of insulin. Therefore, in diglycated insulin, there is the coexistence of one specie glycated at the Nterminals of both chains (Gly1 and Phe1) and another specie containing the two glucitol adducts in chain B (Phe1 and Lys29).

Furthermore, we calculated the relative abundance (RA), in percentage, of glycated peptides for monoglycated and diglycated samples. As normalizing reference 

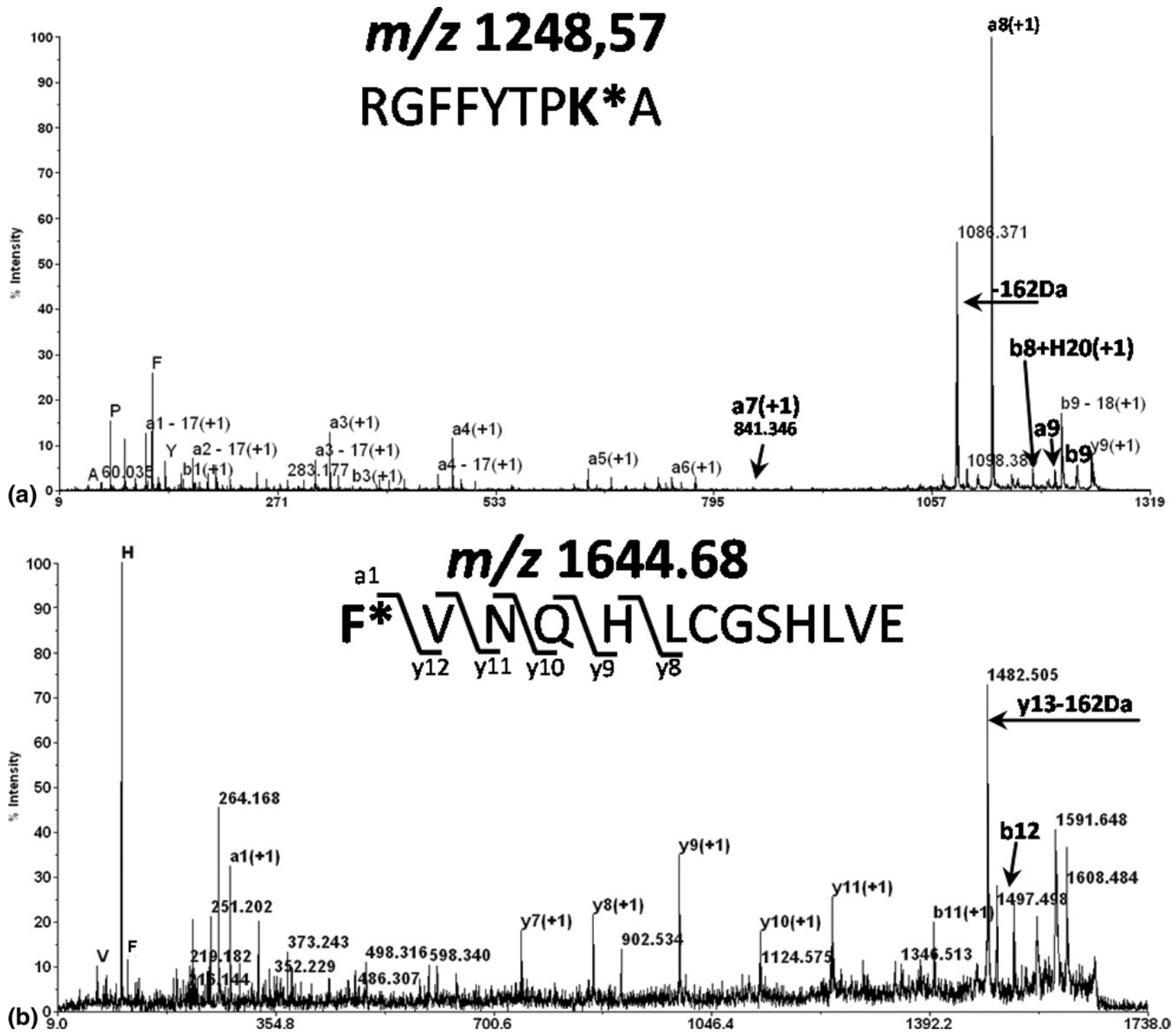

Figure 3. MS/MS spectra of the modified peptide at $\mathrm{m} / \mathrm{z} 1248.57$ (a), corresponding to the sequence between B 22-30 with a glucose adduct located at Lys29, and the modified peptide at $\mathrm{m} / \mathrm{z} 1644.68$ (b), corresponding to the sequence between B 1-13 glycated at Phe1. These glycation sites were found in monoglycated insulin produced under nonreducing conditions.

peak, we used the peak at $\mathrm{m} / \mathrm{z} 1086$ (chain B residues 22-30) since it is the most abundant peak in GluCdigested samples. This analysis indicate that the monoglycated insulin of Figure $1 \mathrm{~b}$ is preferably glycated at Lys29 (Lys29 RA $=30.7 \% \pm 0.8 \%$; Phe- $1 \mathrm{RA}=5.7 \% \pm$ $0.5 \%$ ), while in the monoglycated insulin of Figure 1c, the two possible glycation sites present a similar abundance (Lys29 RA $=6.0 \% \pm 0.1 \%$; Phe1 RA $=5.1 \% \pm 1.3 \%$ ). In the case of diglycated insulin from Figure 1c, although also glycated at Gly1 of chain A, data indicate it appears to be preferably glycated at Lys29 (Lys29 RA $=23.5 \% \pm$ $0.3 \%$; Phe1 RA $=4.6 \% \pm 0.7 \%$ ). Therefore, this diglycated form appears to coexist in two isoforms, one glycated at Phe1 and Lys29 of chain B and the other glycated at Lys29 (chain B) and Gly1 (chain A).
This is the first time that a triglycated form of insulin was isolated and characterized according to its glycation sites. In addition, we were also able to identify in diglycated insulin, the simultaneous presence of two isoforms where three sites of glycation are possible, rather than solely the N-terminals of both chains (Gly1 and Phe1), as reported for human insulin [15]. Therefore, the importance of Lys29 as a possible site of glycation occurring in vivo should not be ignored, as also shown by the results corresponding to the monoglycated insulin produced in nonreducing conditions. To date, the impairment of in vivo and in vitro actions of insulin when glycated has only been studied with insulin glycated under reducing conditions [12, 13, 15], and therefore only considered to be glycated at the N-terminal Phe1 of the B-chain [13] or 


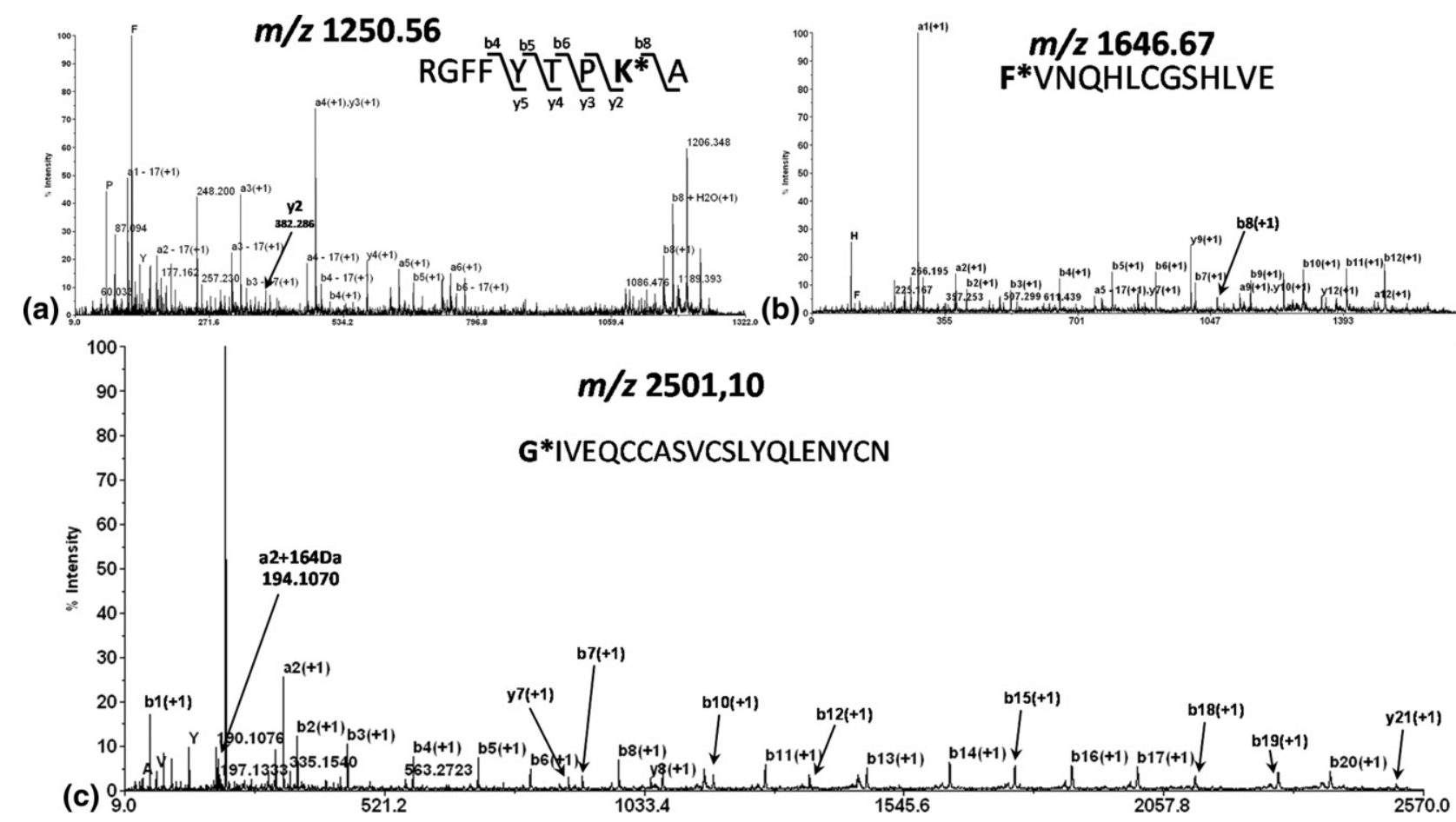

Figure 4. MS/MS spectrum of the modified peptide at $m / z 1250.56$ (a), corresponding to the sequence between B 22-30 with a glucose adduct located at Lys29. (b) Shows the MS/MS spectrum of the modified peptide at $m / z$ 1646.67, which corresponds to the sequence between B 1-13 glycated at Phe1. (c) shows an MS/MS spectrum representative of the A-chain with a single glucitol adduct at $\mathrm{m} / \mathrm{z}$ 2501.10, where the glycation site is located at Gly1. These glycation sites were found in glycated insulin produced under reducing conditions.

glycated at the N-terminals of both chains (Gly1 and Phe1) [15]. It would be of interest to develop further work with glycated insulin at Lys29, since insulin glycation in vivo is associated with characteristic complications of type 2 diabetes such as glucose intolerance and insulin resistance. Elucidating the importance of the structural alterations, which occur in consequence of glycation, in the modulation of insulin action should continue to be of great interest.

\section{Acknowledgments}

The authors express their appreciation for the financial support provided by the "Fundação para a Ciência e Tecnologia" (FCTgrant SFRH/BD/19,769/2004) and PTDC/QUI/68,408/2006.

\section{References}

1. Brownlee, M. Negative Consequences of Glycation. Metabolism. 2000, 49(2) (Suppl 1), 9-13.

2. Bookchin, R. M.; Gallop, P. M. Structure of Hemoglobin AIc: Nature of the N-Terminal beta Chain Blocking Group. Biochem. Biophys. Res. Commun. 1968, 32(1), 86-93

3. Sen, S.; Kar, M.; Roy, A.; Chakraborti, A. S. Effect of Nonenzymatic Glycation on Functional and Structural Properties of Hemoglobin. Biophys. Chem. 2005, 113(3), 289-298.

4. Ganea, E.; Harding, J. J. Inactivation of Glucose-6-Phosphate Dehydrogenase by Glycation. Biochem. Soc. Trans. 1994, 22(4), 445S.

5. Ganea, E.; Harding, J. J. Trehalose and 6-Aminohexanoic Acid Stabilize and Renature Glucose-6-Phosphate Dehydrogenase Inactivated by Glycation and by Guanidinium Hydrochloride. Biol. Chem. 2005, 386(3), 269-278.

6. Takahashi, M.; Lu, Y. B.; Myint, T.; Fujii, J.; Wada, Y.; Taniguchi, N. In Vivo Glycation of Aldehyde Reductase, a Major 3-Deoxyglucosone Reducing Enzyme: Identification of Glycation Sites. Biochemistry. 1995, 34(4), 1433-1148.
7. Blakytny, R.; Harding, J. J. Glycation (Nonenzymic Glycosylation) Inactivates Glutathione Reductase. Biochem I. 1992, 288(Pt 1), 303-307.

8. Niwa, T.; Tsukushi, S. 3-Deoxyglucosone and AGEs in Uremic Complications: Inactivation of Glutathione Peroxidase by 3-Deoxyglucosone. Kidney Int. Suppl. 2001, 78, S37-41.

9. Baldwin, J. S.; Lee, L.; Leung, T. K.; Muruganandam, A.; Mutus, B. Identification of the Site of Nonenzymatic Glycation of Glutathione Peroxidase: Rationalization of the Glycation-Related Catalytic Alterations on the Basis of Three-Dimensional Protein Structure. Biochim. Biophys. Acta. 1995, 1247(1), 60-64.

10. Arai, K.; Iizuka, S.; Tada, Y.; Oikawa, K.; Taniguchi, N. Increase in the Glucosylated Form of Erythrocyte Cu-Zn-Superoxide Dismutase in Diabetes and Close Association of the Nonenzymatic Glucosylation with the Enzyme activity. Biochim. Biophys. Acta. 1987, 924(2), 292-296.

11. Oda, A.; Bannai, C.; Yamaoka, T.; Katori, T.; Matsushima, T.; Yamashita, K. Inactivation of $\mathrm{Cu}, \mathrm{Zn}$-Superoxide Dismutase by In Vitro Glycosylation and in Erythrocytes of Diabetic Patients. Horm. Metab. Res. 1994, 26(1), 1-4.

12. Abdel-Wahab, Y. H.; O'Harte, F. P.; Boyd, A. C.; Barnett, C. R.; Flatt, P. R. Glycation of Insulin Results in Reduced Biological Activity in Mice. Acta Diabetol. 1997, 34(4), 265-270.

13. Boyd, A. C.; Abdel-Wahab, Y. H.; McKillop, A. M.; McNulty, H. Barnett, C. R.; O'Harte, F. P.; Flatt, P. R. Impaired Ability of Glycated Insulin to Regulate Plasma Glucose and Stimulate Glucose Transport and Metabolism in Mouse Abdominal Muscle. Biochim. Biophys. Acta. 2000, 1523(1), 128-134.

14. Dolhofer, R.; Wieland, O. H. Preparation and Biological Properties of Glycosylated Insulin. FEBS Lett. 1979, 100(1), 133-136.

15. O'Harte, F. P.; Boyd, A. C.; McKillop, A. M.; Abdel-Wahab, Y. H.; McNulty, H.; Barnett, C. R.; Conlon, J. M.; Hojrup, P.; Flatt, P. R. Structure, Antihyperglycemic Activity and Cellular Actions of a Novel Diglycated Human Insulin. Peptides. 2000, 21(10), 1519-1526.

16. Schalkwijk, C. G.; Brouwers, O.; Stehouwer, C. D. Modulation of Insulin Action by Advanced Glycation End Products: A New Player in the Field. Horm. Metab. Res. 2008, 40(9), 614-619.

17. Abdel-Wahab, Y. H.; O'Harte, F. P.; Ratcliff, H.; McClenaghan, N. H. Barnett, C. R.; Flatt, P. R. Glycation of Insulin in the Islets of Langerhans of Normal and Diabetic Animals. Diabetes. 1996, 45(11), 1489-1496.

18. Abdel-Wahab, Y. H.; O'Harte, F. P.; Barnett, C. R.; Flatt, P. R. Glycation of Insulin in a Cultured Insulin-Secreting Cell Line. Biochem. Soc. Trans. 1997, 25(1), 128S.

19. Abdel-Wahab, Y. H.; O'Harte, F. P.; Barnett, C. R.; Flatt, P. R. Characterization of Insulin Glycation in Insulin-Secreting Cells Maintained in Tissue Culture. J. Endocrinol. 1997, 152(1), 59-67. 
20. Anzenbacher, P.; Kalous, V. Binding of D-Glucose to Insulin. Biochim. Biophys. Acta. 1975, 386(2), 603-607.

21. Farah, M. A.; Bose, S.; Lee, J. H.; Jung, H. C.; Kim, Y. Analysis of Glycated Insulin by MALDI-TOF Mass Spectrometry. Biochim. Biophys. Acta. 2005, 1725(3), 269-282.
22. McKillop, A. M.; Meade, A.; Flatt, P. R.; O'Harte, F. P. Evaluation of the Site(s) of Glycation in Human Proinsulin by Ion-Trap LCQ Electrospray Ionization Mass Spectrometry. Regul. Pept. 2003, 113(1/3), 1-8.

23. O'Harte, F. P.; Hojrup, P.; Barnett, C. R.; Flatt, P. R. Identification of the Site of Glycation of Human Insulin. Peptides. 1996, 17(8), 1323-1330. 